\title{
Daily interruption of sedative infusions reduced duration of mechanical ventilation and intensive care unit stay in critically ill patients
}

Kress JP, Pohlman AS, O'Connor MF, et al. Daily interruption of sedative infusions in critically ill patients undergoing mechanical ventilation. N Engl J Med 2000 May 18;342:1471-7.

QUESTION: Does daily interruption of sedative infusions in critically ill patients reduce the duration of mechanical ventilation and length of stay in the intensive care unit (ICU) and in hospital?

\section{Design}

Randomised \{allocation concealed\}*, blinded \{patients and clinicians $\} *$ controlled trial with follow up to hospital discharge.

\section{Setting}

A medical ICU in Chicago, Illinois, USA.

\section{Patients}

150 patients who were intubated and receiving mechanical ventilation and who required sedation by continuous intravenous (IV) infusion (eg, patients who showed agitation or discomfort after recovering from drugs used to facilitate intubation). Exclusion criteria were pregnancy, transfer from an outside institution where sedatives had already been given, and admission after resuscitation from cardiac arrest. 128 patients (53\% women) were included in the final analysis (22 patients died or were extubated on the first or second day in the ICU).

\section{Intervention}

75 patients were allocated to daily interruption of sedative infusion beginning 48 hours after enrolment; infusions were interrupted until the patient was awake and could follow instructions or became uncomfortable or agitated. 75 patients were allocated to continuous infusion of sedatives, with interruption only at the discretion of the intensive care team. Within each group, the sedative infusion comprised morphine (initial IV bolus of $2-10 \mathrm{mg}$ as needed and continuous infusion at $1-5 \mathrm{mg} / \mathrm{h}$ ) plus a randomly assigned, non-analgesic sedative: midazolam (initial IV bolus of $0.5-5 \mathrm{mg}$ every $1-5 \mathrm{~min}$ as needed and continuous infusion at $1-2 \mathrm{mg} / \mathrm{h}$, increased in increments of $1-2 \mathrm{mg} / \mathrm{h}$ until adequate sedation achieved) or propofol (continuous infusion at $5 \mu \mathrm{g} / \mathrm{kg}$ body weight/ min, increased in increments of $5-10 \mu \mathrm{g} / \mathrm{kg} / \mathrm{min}$ every 2 min until adequate sedation achieved).

\section{Main outcome measures}

Main outcomes were duration of mechanical ventilation, length of ICU stay, and length of hospital stay. Other outcomes included percentage of days of wakefulness, use of neurological tests, adverse events (eg, patient removal of endotracheal tube), reintubation, tracheostomy, transfer to facility for long term ventilation, discharge to home, or in hospital mortality.

\section{Main results}

Analysis was by intention to treat. Patients who received daily interruption of sedative infusions spent a median of 2.4 fewer days on mechanical ventilation $(4.9 \vee 7.3 \mathrm{~d}$, $\mathrm{p}=0.004)$ and 3.5 fewer days in the ICU $(6.4 v 9.9 \mathrm{~d}$, $\mathrm{p}=0.02)$ than patients who received continuous sedative infusions; the groups did not differ for total length of hospital stay. The daily interruption group had a higher percentage of days of wakefulness $(86 \% v 9 \%, \mathrm{p}<0.001)$ and fewer tests to assess changes in mental status $(p=0.02)$. The groups did not differ for other outcomes. Conclusion

Daily interruption of continuous sedative infusion in critically ill patients reduced duration of mechanical ventilation and intensive care unit stay, but not duration of total hospital stay.

*Information provided by author.

\section{COMMENTARY}

Sedation is often required during critical illness to facilitate mechanical ventilation, reduce metabolic rate, and promote patient comfort. Continuous sedative infusions can make differentiation between adequate and excessive sedation difficult. Excessive sedation may prolong the duration of mechanical ventilation and interfere with the ability to assess neurological status, thereby increasing healthcare costs and risk of complications. Currently, there is limited evidence to guide sedation practice. The study by Kress $e t a l$ is one of the first attempts to evaluate the effects of daily interruption of continuous infusions on the duration of mechanical ventilation and length of stay.

The participants were ventilated medical ICU patients. Although duration of mechanical ventilation was an important outcome, there was no report of a standardised weaning protocol. Also, clinicians may not have been blinded to the research protocol despite attempts to limit their awareness. Any differences attributable to bias or weaning variations were most likely minimised through random assignment to groups. Of the 60 participants in the control group, 18 had infusions temporarily stopped during the study. Frequency was not described; frequent stoppages might have decreased the difference found between groups.

These findings are relevant to nurses caring for mechanically ventilated patients who require continuous sedation. Daily wakening should be attempted, unless contraindicated. This intervention may also reduce the total drug dose and risk of oversedation, especially when using morphine or midazolam. Patients in the intervention group on midazolam received less midazolam (229.8 $v 425.5 \mathrm{mg}, \mathrm{p}=0.05)$ and morphine (205 $\mathrm{mg} v 481 \mathrm{mg}, \mathrm{p}=0.009$ ) than patients in the control group; for patients on propofol, the groups did not differ in the amount of propofol or morphine received. Yet, patients were awake on $>80 \%$ of days in both subgroups of the intervention group, and this percentage did not differ according to sedative used. Also, no differences in primary outcomes were found when patients were grouped by type of sedative.

Potential adverse effects of abrupt arousal (eg, bronchospasm, myocardial ischaemia, intracranial hypertension, or panic) were not measured; caution should be exercised to establish individual patient suitability. Further studies are needed to contrast bolus dosing to intermittent interruption of continuous sedation.

Brenda Lynn Morgan, RN, CNCC(C), BScN Clinical Educator, Critical Care Trauma Centre London Health Sciences Centre London, Ontario, Canada 